\title{
Frustrated magnets in three dimensions: a nonperturbative approach
}

\author{
B. Delamotte†, D. Mouhanna† and M. Tissier $\ddagger$ \\ † Laboratoire de Physique Théorique et Hautes Energies \\ Université Paris VI-Pierre et Marie Curie, Université Paris VII-Denis Diderot \\ and UMR 7589 of CNRS, 2 Place Jussieu, 75251 Paris Cedex 05, France \\ $\ddagger$ Laboratoire de Physique Théorique des Liquides \\ Université Paris VI-Pierre et Marie Curie and UMR 7600 of CNRS, 4 Place \\ Jussieu, 75252 Paris Cedex 05, France
}

\begin{abstract}
.
Frustrated magnets exhibit unusual critical behaviors: they display scaling laws accompanied by nonuniversal critical exponents. This suggests that these systems generically undergo very weak first order phase transitions. Moreover, the different perturbative approaches used to investigate them are in conflict and fail to correctly reproduce their behavior. Using a nonperturbative approach we explain the mismatch between the different perturbative approaches and account for the nonuniversal scaling observed.

PACS numbers: 75.10.Hk, 64.60.-i, 11.10.Hi
\end{abstract}

\section{Introduction}

After twenty five years of investigations, the nature of the phase transition undergone by frustrated magnets is still strongly debated [1, 2. The main feature of these systems is that, due to frustration, their ground state displays a noncollinear order. This is the case for the celebrated Stacked Triangular Antiferromagnets (STA) whose ground state exhibits a $120^{\circ}$ structure. As a consequence, for $N=3$ for instance, the $S O(3)$ symmetry group of the high-temperature phase is fully broken in the lowtemperature one. Also, the order parameter is no longer given by a vector, as in the nonfrustrated case, but by a matrix made of two orthonormal vectors $\vec{\phi}_{1}$ and $\vec{\phi}_{2}$. These characteristics have led to a conjecture of the existence of a new universality class for frustrated magnets. In fact, the interpretation as well as the description of their critical physics appear to be considerably more involved than for nonfrustrated magnets: first, because there are several experimental facts incompatible with a universal critical behavior; second, because the different theoretical approaches used to analyze their behaviors are in conflict and fail to reproduce the experimental data.

\section{Experimental and numerical contexts}

The first indications of a nontrivial critical behavior for frustrated magnets appear in the analysis of the experimental and numerical data. An extensive analysis of the compounds and systems supposed a priori to belong to the same universality class as 
the STA, such as Helimagnets, has been performed by the present authors in [3, 4]. Without entering into the details we give the main features that have emerged from this analysis. First, scaling laws are found in most compounds. This is also the case within all - but an important one 5] - numerical simulations of the STA. This scaling behavior is associated with critical exponents that differ from those of the standard $O(N) / O(N-1)$ universality classes. This fact has been at the origin of the belief that frustrated magnets could constitute a new universality class. However, there are other facts characterizing the critical behavior of both XY and Heisenberg systems that go against this belief.

i) There are two groups of compounds that clearly differ by their critical exponents.

ii) The anomalous dimension $\eta$ is negative for many compounds and for many systems numerically simulated. We recall that the anomalous dimension $\eta$ is always positive if the phase transition is truly of second order and if the underlying field theory is a Ginzburg-Landau-Wilson (GLW) $\phi^{4}$-like theory, as it is the case here.

iii) The scaling relations are violated by several standard deviations for many compounds.

iv) Models differing from STA by their microscopical details undergo either strong first order phase transitions (XY case) [5] 6] or exhibit scaling behaviors incompatible with that of the numerical STA model (Heisenberg case) 17, 8, 9.

v) Recent Monte Carlo and Monte Carlo RG approaches of STA and GLW models give clear indications of first order behaviors [5].

From all these observations, it appears that an explanation of the critical physics of frustrated magnets assuming a standard second order critical behavior relying on the existence of a unique universality class is very likely excluded.

\section{Field theoretical context}

\subsection{The nonlinear sigma model approach}

A first theoretical approach consists in a low-temperature expansion performed near $d=2$ on the suitable field theory, the $O(3) \times O(2) / O(2)$ nonlinear sigma (NL $\sigma$ ) model [10, 11] which is obtained by expressing the interaction between spins in terms of the Goldstone modes. The renormalization of the interaction between these modes is treated within a double expansion in the temperature $T$ and in $\epsilon=d-2$. The result of this study is that there exists, above $d=2$, a stable fixed point 10, 11]. At this fixed point, the symmetry breaking scheme is enlarged from $O(3) \times O(2) / O(2)$ to $O(3) \times O(3) / O(3)$ which itself has the same local structure as $O(4) / O(3)$ on which is built the $\mathrm{NL} \sigma$ model relevant to four-component nonfrustrated spins. As seen from the NL $\sigma$ model approach - that only depends on this local structure - the two above theories are equivalent to all orders. Accordingly, the critical properties of the STA model between $d=2$ and $d=4$ should be governed by the standard $O(4) / O(3)$ universality class 10, 11. This is, however, in disagreement with both the experimental and numerical data as well as the weak-coupling analysis of the GLW model of frustrated magnets around $d=4$. Therefore, there must exist nonperturbative phenomena - with respect to the expansion parameters $T$ and $\epsilon=d-2$ - leading to the breakdown of the NL $\sigma$ model approach somewhere between $d=2$ and $d=3$. 


\subsection{The GLW model approach}

An alternative approach is to consider a Ginzburg-Landau-Wilson (GLW) representation of the physics of frustrated magnets [12, 13, 14, 15, 16]. This can be obtained by relaxing, via a potential, the constraints defining the ground state. The resulting Hamiltonian writes:

$$
\begin{aligned}
H=\int d^{d} x & \left\{\frac { 1 } { 2 } \left(\left(\partial \overrightarrow{\phi_{1}}\right)^{2}+\left(\partial{\overrightarrow{\phi_{2}}}^{2}\right)+\frac{m^{2}}{2}\left({\overrightarrow{\phi_{1}}}^{2}+{\overrightarrow{\phi_{2}}}^{2}\right)+\right.\right. \\
& +u\left({\overrightarrow{\phi_{1}}}^{2}+{\overrightarrow{\phi_{2}}}^{2}\right)^{2}+v\left({\overrightarrow{\phi_{1}}}^{2}{\overrightarrow{\phi_{2}}}^{2}-\left(\overrightarrow{\phi_{1}} \cdot{\overrightarrow{\phi_{2}}}^{2}\right)\right\} .
\end{aligned}
$$

The RG equations for the coupling constants $u$ and $v$ have been first determined in a double expansion in $u, v$ and $\epsilon=4-d$. At leading order [13, 14, 15], they display no stable fixed point in the XY and Heisenberg cases. A first order phase transition is thus expected in $d=3$, in contradiction with the NL $\sigma$ model results and with the existence of scaling behaviors for frustrated magnets. An important generalization of the GLW model (11) is to consider $\vec{\phi}_{1}$ and $\vec{\phi}_{2}$ as $N$-component vectors [13, 14, 15]. For $N$ larger than a critical value $N_{c}(d)$ depending on the dimension, the RG equations display two nontrivial fixed points: one, $C_{+}$, is stable; the other one, $C_{-}$, is unstable. Above $N_{c}(d)$, the transition is thus predicted to be of second order. As $N$ is lowered starting from values of $N>N_{c}(d)$, the two fixed points $C_{+}$and $C_{-}$get closer and finally collapse together for $N=N_{c}(d)$. Below $N_{c}(d)$, there is no longer a stable fixed point and the transition is expected to be of first order. The value of $N_{c}(d)$ has been computed by several perturbative means: in a double expansion in $u, v$ and $\epsilon=4-d$ up to five-loops [17 and directly in $d=3$ in a weak-coupling expansion up to six-loops [18. These computations lead to an almost common value $N_{c}(d=3) \simeq 6$. This result could be interpreted as the existence of a first order phase transition in $d=3$ for both XY and Heisenberg spins. This is only true within the $4-\epsilon$ expansion. In effect - and rather surprisingly - within the six-loop weak-coupling expansion performed in $d=3$ by Pelissetto et al [18, a fixed point is found for $N=2$ and $N=3$. It has been conjectured by Calabrese et al [19] 20] that these fixed points could explain the spreading of critical exponents encountered in frustrated magnets. Indeed, due to the specific structure of the RG flow around the focus fixed point, the critical exponents display strong variations along the RG trajectories that could explain the lack of universality observed experimentally and numerically. Let us now underline several drawbacks of the scenario of Calabrese et al. First, it is based on the existence of fixed points that are not related to any already known fixed points. In particular, the fixed points found for $N=2$ and $N=3$ within this computation in $d=3$ that would govern the critical physics of frustrated magnets are, according to Pelissetto et al and Calabrese et al, nonanalytically related to those found in the large$N$ as well as in the $4-\epsilon$ expansions. This means that there is no way to check their existence using these perturbative methods. Second, it is very difficult to account, in this framework, for the first order behavior deduced from several numerical simulations of XY and Heisenberg systems. Third, it is also very difficult to explain why there is no physical system characterized by the exponents associated with the fixed point found by Pelissetto et al. Finally, there is no explanation of the breakdown of the NL $\sigma$ model predictions. 


\section{Effective average action approach}

To clarify this situation we have employed a nonperturbative approach based on the concept of effective average action [21, 22], $\Gamma_{k}[\phi]$, which is a coarse grained free energy in which only fluctuations with momenta $q \geq k$ have been integrated out. The field $\phi$ stands here for an average order parameter at the running scale $k$. In the limit $k \rightarrow 0$, all modes are taken into account and $\Gamma_{k=0}$ identifies with the usual Gibbs free energy, i.e. the effective action $\Gamma$. At the scale $k=\Lambda$ - the overall cut-off - no fluctuation has been integrated and $\Gamma_{k=\Lambda}$ identifies with the microscopic Hamiltonian. The $k$-dependence of $\Gamma_{k}$ is controlled by an exact evolution equation 23, 24]:

$$
\frac{\partial \Gamma_{k}}{\partial t}=\frac{1}{2} \operatorname{Tr}\left\{\frac{\partial R_{k}}{\partial t} \frac{1}{\Gamma_{k}^{(2)}+R_{k}}\right\},
$$

where $t=\ln k / \Lambda$. The trace has to be understood as a momentum integral as well as a summation over internal indices. In equation (2), $\Gamma_{k}^{(2)}$ is the exact field-dependent inverse propagator - i.e. the second derivative of $\Gamma_{k}$ - and $R_{k}$ is the effective infrared cut-off which suppresses the propagation of modes with momenta $q<k$. The effective average action $\Gamma_{k}$ is a functional, invariant under the symmetry group of the system and thus includes all powers of all invariants - and their derivatives - built from the average order parameter. Thus, equation (2) is a nonlinear functional equation, too difficult to solve exactly so that $\Gamma_{k}$ must be truncated. One - fruitful - possibility is to perform a double expansion of $\Gamma_{k}$ in derivatives and in fields keeping a finite number of monomials in the fields while including the most relevant derivative terms 22 . We have first considered the following truncation $3,4,25]$ :

$$
\begin{aligned}
\Gamma_{k}\left[\vec{\phi}_{1}, \vec{\phi}_{2}\right]= & \int d^{d} x\left\{\frac{Z_{k}}{2}\left(\left(\partial \vec{\phi}_{1}\right)^{2}+\left(\partial \vec{\phi}_{2}\right)^{2}\right)+\right. \\
& \left.\frac{\omega_{k}}{4}\left(\vec{\phi}_{1} \cdot \partial \vec{\phi}_{2}-\vec{\phi}_{2} \cdot \partial \vec{\phi}_{1}\right)^{2}+\frac{\lambda_{k}}{4}\left(\frac{\rho}{2}-\kappa_{k}\right)^{2}+\frac{\mu_{k}}{4} \tau\right\}
\end{aligned}
$$

where $\left\{\omega_{k}, \lambda_{k}, \kappa_{k}, \mu_{k}, Z_{k}\right\}$ are the coupling constants that parametrize the evolution of the model with the scale, while $\rho=\operatorname{Tr}{ }^{t} \phi \phi$ and $\tau=\frac{1}{2} \operatorname{Tr}\left({ }^{t} \phi \phi\right)^{2}-\frac{1}{4}\left(\operatorname{Tr}^{t} \phi \phi\right)^{2}$ are the two independent $O(N) \times O(2)$ invariants built from the average order parameter $\phi=\phi_{a b}$, a real $N \times 2$ matrix that gathers the two vectors $\vec{\phi}_{1}$ and $\vec{\phi}_{2}$. This ansatz realizes the symmetry breaking scheme of frustrated magnets: $O(N) \times O(2) \rightarrow$ $O(N-2) \times O(2)_{\text {diag }}$. We have derived the nonperturbative flow equations for the different coupling constants using equations (2) and (31). We have also checked the convergence of our results with respect to the order of the truncation of $\Gamma_{k}$ in $\rho$ and $\tau$. The explicit recursion equations for the coupling constants are too lengthy to be displayed here [3, 4, 25] so we concentrate on the physical results.

\subsection{Fixed point structure}

We have numerically analyzed our flow equations by varying the dimension between $d=2$ and $d=4$ for different values of $N$, identified the different fixed points and studied their stability. Around $d=2$ we find, for all $N>2$, a stable fixed point $C_{+}$ which identifies with the NL $\sigma$ model fixed point. For sufficiently large values of $N-$ $N>21.8$ - one can follow this fixed point up to $d=4$ where the one-loop results of the weak-coupling expansion performed around this dimension are recovered. We are thus able to smoothly interpolate between the perturbative results obtained in $d=2$ 
and $d=4$. On the other hand, for any value of $N>2$, increasing $d$, one finds a new and unstable fixed point $C_{-}$in a dimension $d_{1}(N)$. For any value of $N<21.8$ and as $d$ is further increased, the two fixed points $C_{+}$and $C_{-}$get closer, coalesce and finally become complex in a dimension $d_{2}(N)$. Above this dimension $d_{2}(N)$, no real fixed point is found, a fact which is interpreted as the sign of a first order behavior. This collapse of the fixed points $C_{+}$and $C_{-}$, for different values of $N$, generates the curve $N_{c}(d)$. As already said, for large values of $N$ - typically $N$ of order $21.8-$ the collapse occurs around $d=4$ in agreement with the — standard - weak-coupling analysis performed around this dimension. However, for low values of $N-N=3$ for instance - this collapse of fixed points operates between $d=2$ and $d=3$ : one has $d_{2}(N=3) \simeq 2.8$. This means that our approach provides a nonperturbative solution to the breakdown of the NL $\sigma$ model approach [3, 25, 26]: the stable $O(4) / O(3)$ fixed point obtained within this approach collapses in $d \simeq 2.8$ with another - unstable fixed point which is not seen in a low-temperature expansion.

\subsection{The physics in $d=3$}

We now give the results we have obtained in $d=3$ within our approach [3, 4]. We have computed the value of $N_{c}(d)$ in this dimension and found $N_{c}(d=3)=5.1$. Then, we have searched fixed points below this value of $N_{c}$ in order to test the results of Pelissetto et al and the conjecture of Calabrese et al that the physics of frustrated magnets would be governed by fixed points living in the region $N<N_{c}(d=3)$. This search has been unsuccessful. In fact, within our approach, we have discovered that, although there is no fixed point, there exists a whole region in the space of coupling constants in which the RG flow is slow. This generalizes the notion of pseudo-fixed point introduced by Zumbach [27]. For the systems corresponding to these coupling constants, the correlation lengths are very large at the transitions, that are thus very weakly of first order. We have found scaling behaviors on large ranges of temperature for both XY and Heisenberg magnets (see figure (10). Moreover, we have been able to account for the spreading of (pseudo-)critical exponents that characterize these systems and, by varying the initial conditions of the RG flow, to reproduce the critical exponents of the numerically simulated systems and of many compounds. For XY systems, we have found $0.25<\beta<0.38$ and $0.47<\nu<0.58$ and, for Heisenberg systems, $0.27<\beta<0.42$ and $0.56<\nu<0.71$, ranges of values in which almost all experimental and numerical values enter. Let us give more details. We first consider the XY case. According to the experimental values of the critical exponents, the compounds split into two groups: STA compounds $-\mathrm{CsMnBr}_{3}, \mathrm{CsNiCl}_{3}$ and $\mathrm{CsMnI}_{3}$ - for which one has, on average, $\beta=0.237(4), \nu=0.555(21), \alpha=0.344(5)$ and $\gamma=1.075(42)$, and Helimagnets compounds - Holmium and Dysprosium for which one has $\beta=0.389(7), \nu=0.558(25)$ and $\gamma=1.10(5)$ ( $\alpha$ is not given since it is poorly determined). Within our computations, we find initial conditions of the flow leading to critical exponents close to those of Helimagnets: $\beta=0.38$, $\nu=0.58$ and $\gamma=1.13$. We also easily find initial conditions leading to $\beta=0.25$, corresponding essentially to STA compounds. For such values of $\beta$, we find that $\nu$ varies between 0.47 and 0.49 , which is somewhat below the value found for $\mathrm{CsMnBr}_{3}$. The same splitting of the compounds happens in the Heisenberg case. There is a group composed of $\mathrm{Cu}(\mathrm{HCOO})_{2} 2 \mathrm{CO}\left(\mathrm{ND}_{2}\right)_{2} 2 \mathrm{D}_{2} \mathrm{O}, \mathrm{Fe}\left[\mathrm{S}_{2} \mathrm{CN}\left(\mathrm{C}_{2} \mathrm{H}_{5}\right)_{2}\right]_{2} \mathrm{Cl}, \mathrm{VCl}_{2}$ and $\mathrm{VBr}_{2}$ characterized by $\beta=0.230(8), \alpha=0.272(35), \nu=0.62(5)$ and $\gamma=1.105(21)$. There is also a group made of $\mathrm{CsNiCl}_{3}, \mathrm{CsMnI}_{3}$ and $\mathrm{CsMn}\left(\mathrm{Br}_{0.19}, \mathrm{I}_{0.81}\right)_{3}$ for which 

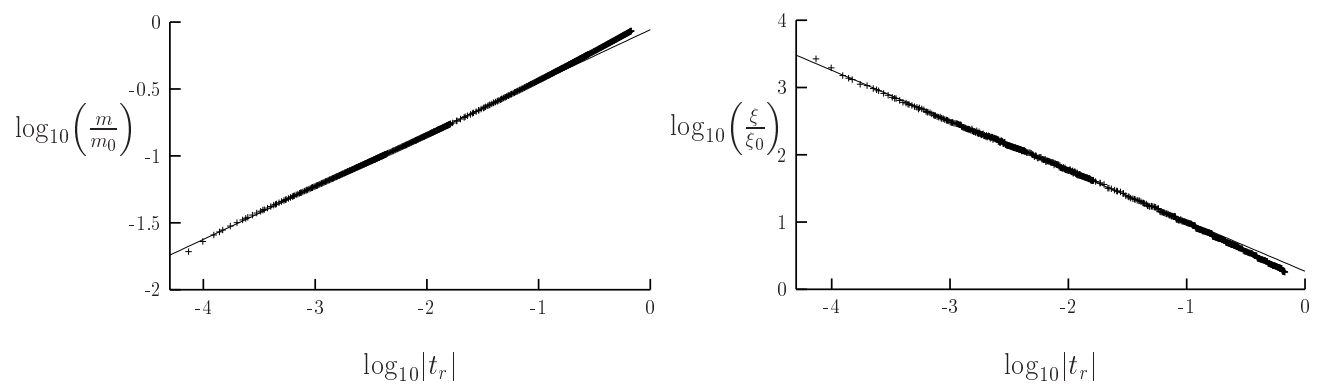

Figure 1. Log-log plot of the order parameter $m$ and of the correlation length $\xi$ for $N=3$ as functions of the reduced temperature $t_{r}$.

$\beta=0.287(9), \alpha=0.243(3), \nu=0.585(9)$ and $\gamma=1.181(33)$. Within our computations one can find $\beta=0.27$ and $\nu=0.56$ which are close to the experimental values of the second group. In contrast, we have not been able to reproduce correctly the critical exponents of the first group. This difficulty to reproduce the critical exponents originates in the different approximations we have used, in particular the truncations in fields and derivatives. Beyond this technical difficulty, the trouble is that, once the universality is lost, the pseudo-critical exponents depend on the specific compound considered and their precise determination would require to know precisely the microscopic structure of the compounds or systems studied - providing the initial conditions of the RG flow - and to take into account the full field-dependence of the potential in $\Gamma_{k}$. Finally and interestingly, we have also been able to find, in the Heisenberg and in the XY cases, initial conditions leading to pseudo-critical exponents in good agreement with those obtained within the six-loop calculation [18. In the Heisenberg case, one can find exactly the same values $-\beta=0.30, \nu=0.55$ and $\gamma=1.06$ - than Pelissetto et al [18]. In the XY case, one finds $\beta=0.33, \nu=0.56$ and $\gamma=1.07$ which are very close to the six-loop results: $\beta=0.31(2), \nu=0.57(3)$ and $\gamma=1.10(4)$.

\section{Conclusion}

On the basis of their specific symmetry breaking scheme, it has been proposed that the critical physics of frustrated systems in $d=3$ could be characterized by critical exponents associated with a new universality class. From this point of view, the study of frustrated magnets has been rather disappointing, the experimental and numerical contexts excluding such an hypothesis. At the same time, the phenomenology of frustrated magnets has displayed a novel kind of critical behavior: the existence of generic scaling without universality 3. 4. Within the framework of our nonperturbative approach, this generic and nonuniversal scaling finds a natural explanation in terms of slowness of the flow. This method has also explained the mismatch between the different perturbative approaches by means of a mechanism of annihilation of fixed points that invalidates the low-temperature perturbative approach performed on the NL $\sigma$ model. It would be satisfying to understand the very origin of this failure. The influence of nontrivial topological configurations on the phase transition in $d=3$ has been invoked. It remains however to confirm that these configurations indeed play such a crucial role and to understand whether they are really responsible for the first order character of the transitions. Finally, and even though 
it could appear as a rather formal question, it would be also satisfying to clarify the discrepancy between the nonperturbative and the six-loop perturbative approaches. The matching between the sets of exponents found within these different approaches suggests that there exists a common origin to these two sets of exponents. One can easily imagine that, while the two kinds of computations would be almost converged as for the determination of the exponents themselves, one of these computations would not be converged as for the true nature - real or complex - of the fixed points. This question will be likely clarified in the near future.

Note added in proof. Let us mention that a Monte Carlo study [28] has confirmed the existence of first order behaviors for a whole family of XY frustrated magnets, in agreement with our scenario.

\section{References}

[1] H. T. Diep, editor. Magnetic systems with competing interactions. World Scientific, 1994.

[2] H. Kawamura. J. Phys.: Condens. Matter, 10:4707, 1998.

[3] M. Tissier, B. Delamotte, and D. Mouhanna. Phys. Rev. B, 67:134422, 2003.

[4] B. Delamotte, D. Mouhanna, and M. Tissier. cond-mat/0309101

[5] M. Itakura. J. Phys. Soc. Jap., 72:74, 2003.

[6] D. Loison and K. D. Schotte. Euro. Phys. J. B, 5:735, 1998.

[7] D. Loison and K. D. Schotte. Euro. Phys. J. B, 14:125, 2000.

[8] H. Kunz and G. Zumbach. J. Phys. A: Math. Gen., 26:3121, 1993.

[9] D. Loison. Physica A, 275:207, 1999.

[10] P. Azaria, B. Delamotte, and T. Jolicœur. Phys. Rev. Lett., 64:3175, 1990.

[11] P. Azaria, B. Delamotte, F. Delduc, and T. Jolicœur. Nucl. Phys. B [FS], 408:485, 1993.

[12] D. R. T. Jones, A. Love, and M. A. Moore. J. Phys. C: Solid St. Phys., 9:743, 1976.

[13] T. Garel and P. Pfeuty. J. Phys. C: Solid St. Phys., 9:L245, 1976.

[14] D. Bailin, A. Love, and M. A. Moore. J. Phys. C: Solid State Phys., 10:1159, 1977.

[15] M. Yosefin and E. Domany. Phys. Rev. B, 32:1778, 1985.

[16] H. Kawamura. Phys. Rev. B, 38:4916, 1988.

[17] P. Calabrese and P. Parruccini. cond-mat/0308037

[18] A. Pelissetto, P. Rossi, and E. Vicari. Phys. Rev. B, 63:140414, 2001.

[19] P. Calabrese, P. Parruccini, and A. I. Sokolov. Phys. Rev. B, 66:180403, 2002.

[20] P. Calabrese, P. Parruccini, and A. I. Sokolov. Phys. Rev. B, 68:094415, 2003.

[21] C. Wetterich. Nucl. Phys. B, 352:529, 1991.

[22] J. Berges, N. Tetradis, and C. Wetterich. Phys. Rep., 363:223, 2002.

[23] C. Wetterich. Phys. Lett. B, 301:90, 1993.

[24] N. Tetradis and C. Wetterich. Nucl. Phys. B [FS], 422:541, 1994.

[25] M. Tissier, B. Delamotte, and D. Mouhanna. Phys. Rev. Lett., 84:5208, 2000.

[26] M. Tissier, D. Mouhanna, and B. Delamotte. Phys. Rev. B, 61:15327, 2000.

[27] G. Zumbach. Phys. Rev. Lett., 71:2421, 1993.

[28] A. Peles, B.W. Southern, B. Delamotte, D. Mouhanna and M. Tissier cond-mat/0401259 